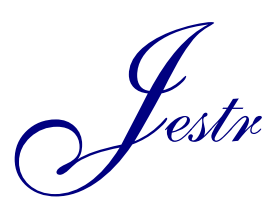

\title{
PMU placement methodology for voltage stability monitoring in Electrical Power Systems
}

\author{
Sindy L. Ramírez P*, Gladys Caicedo D and Carlos Arturo Lozano M \\ School of Electrical and Electronic Engineering, High Voltage Research Group, Universidad del Valle.
}

Received 18 July 2018; Accepted 14 September 2019

\begin{abstract}
This article presents a PMU placement methodology for voltage stability monitoring in Electrical Power Systems (EPSs). This methodology identifies the nodes and areas that are most sensitive to voltage stability problems, to locate the PMUs and to guarantee the voltage stability monitoring. Unlike other studies, this methodology considers the EPS as a whole and under N-1 contingencies. Criteria such as voltage stability margins, V-Q sensitivity analysis, modal analysis with participation factors in nodes, lines and generators under normal operating conditions and critical contingencies were used to identify the nodes to be installed with the PMUs. The methodology was applied to the New England 39-node test system, and the results show that this methodology is appropriate for the identification of areas and nodes that are sensitive to voltage stability problems and that these are the EPS points that should be considered for the installation of the PMUs.
\end{abstract}

Keywords: Phasor Measurement Unit (PMU), Optimal PMU Placement OPP, voltage stability monitoring.

\section{Introduction}

Recent measurement technologies using phasor measurement units have allowed the use of synchronized phasor information for different applications such as stability monitoring, control and protection against EPS faults [1]. The first step in the use of phasor technology through PMUs is their placement in the EPS, to observe the network and the use of information for different applications. The problem of Optimal PMU Placement (OPP) is to determine the amount and placement of the transmission system, in which the meters should be installed to minimize the investment costs for the measurement equipment and, thus, to reinforce the information system used in the EPS analysis. Therefore, it is important to develop a useful and convenient PMU placement strategy with a limited number of PMUs.

Topological and numerical methods have been developed to determine the strategy of optimal PMU placements according to their specific applications [2]. An approach based on a numerical observation uses the Jacobian matrix, which reflects the configuration of the system and the set of measurements. On the other hand, numerical methods involve large manipulations of the network matrix and are computationally costly. There are many optimal PMU placement techniques based on the concept of numerical observability, such as the Simulated Annealing method [3], the Tabu Search method [4], [5], [6], and Genetic Algorithms [7], [8]. However, all of these techniques, because they are iterative in nature, require a longer convergence time, and their convergence depends on their initial condition. On the other hand, approaches based on topological observability use graph theory to find optimal placements to make the system topologically observable. The methods that are based on this

*E-mail address: sindy.lorena.ramirez@correounivalle.edu.co ISSN: 1791-2377 @ 2019 School of Science, IHU. All rights reserved. doi:10.25103/iestr.126.14 concept include Depth First Search [9], [10], Spanning Tree [11] and the Integer Linear Programming [12], [13].

Some studies [1], [8], [14] and [15] consider N-1 contingencies of output lines, and other studies consider a single line output or the loss of a single PMU, such as [16], [15], [17]. Regarding the voltage stability in [1], the OPP is applied under normal operating conditions and critical contingencies. Similarly, in [14], a method that develops the voltage stability load index (VSLI) for an EPS using the data of optimally placed PMUs is presented. The optimal PMU placement is made for island operating conditions.

On the other hand, in [3], the sensitivity concept is introduced to OPP. The sensitivities of the node and line parameters are considered during the OPP search for a complete observability of the EPS. The method uses the Simulated Annealing algorithm, in which the problem is to minimize the discrete objective function for a restriction in which the EPS is topologically observable, and the PMUs are placed in the nodes that have higher sensitivities.

However, an analysis of the placement algorithms reveals that in all of the methods mentioned above, the PMU placements were conducted while assuming that the EPS is in a normal operating state and without considering the relevant criteria for the voltage stability monitoring that includes the $\mathrm{N}-1$ contingencies. The criteria defined and used in this methodology are V-Q sensitivity analysis, voltage stability margin, and modal analysis with participation factors in nodes, lines and generators. This study presents a PMU placement methodology for stability monitoring that considers all of the points mentioned above for both normal operations and EPS topological changes. The methodology also considers critical contingencies to the voltage stability problem and the most sensitive nodes to the EPS voltage stability problems. The proposed methodology for PMU placement has been tested in the New England 39-node system. The results show that the proposed method guarantees the inclusion of relevant voltage stability criteria not only 
under normal operating conditions but also in critical contingency cases.

\section{Theoretical Framework}

The application in this study is voltage stability monitoring using PMU information. The placement criteria for the PMU placement methodology are as follows:

\subsection{V-Q Sensitivity analysis}

This criterion is based on analyzing the voltage variation with respect to the power injection in the nodes of the system. The analysis allows us to identify the most sensitive nodes when changing reactants.

When the system is stable, the V-Q sensitivity is positive, and when it is unstable, it is negative. Small positive values of sensitivity represent higher system stability, whereas large values indicate that the stability limit is be approached [18].

\subsection{Voltage stability margin}

The voltage stability margin is obtained by varying the active power and studying the behavior of the voltages of the nodes. Different "V-P" curves can be plotted, which change the load power factor and the node voltage "i" [18]. To make these graphs in large power systems, conventional power flows or progressive power flows can be used. These flows calculate the voltage variations before the increase in the loads. The load variation is made by increasing the power from an initial value until finding the power transfer limit to the node "i". The node voltage variation is plotted to analyze the changes in the responses to an increase in the load, as shown in Error! Reference source not found.

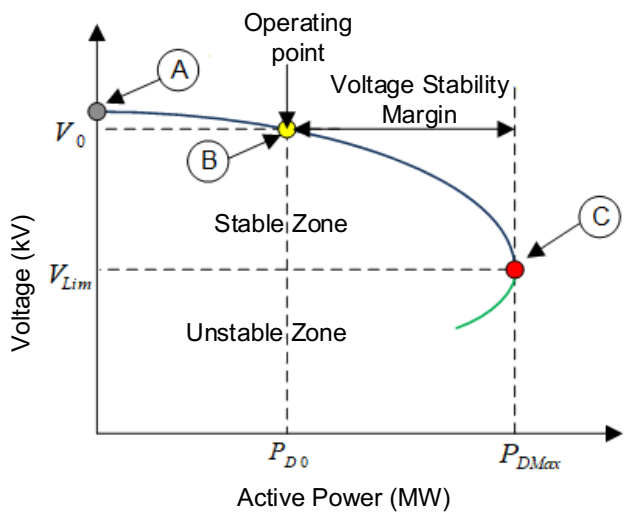

Fig. 1. "V-P" Curve in node "i" of the EPS.

Where:

$\mathrm{P}_{\mathrm{D} 0}$ : Active power supplied to node " $\mathrm{i}$ " at the operating point $\mathrm{P}_{\mathrm{DMax}}$ : Maximum active power supplied to node " $\mathrm{i}$ "

$\mathrm{V}_{0}$ : Voltage of node "i" at the operating point

$\mathrm{V}_{\text {Lim: }}$ : Voltage limit of node "i" to maintain the stability

Point " $\mathrm{A}$ " in the curve corresponds to the operating point without a load, and as the load increases, the voltage decreases. Point " $\mathrm{B}$ " corresponds to the operating point of the system, in which the active power consumption is equal to " $\mathrm{P}_{\mathrm{D} 0}$ " and its operating voltage is " $\mathrm{V}_{0}$ ". Point " $\mathrm{C}$ " corresponds to the active power transfer limit " $P_{\text {Dmax }}$ ", in which the voltage level is equal to " $\mathrm{V}_{\mathrm{Lim}}$ ". The voltage stability margin is calculated as the difference between the maximum load "P $P_{\text {DMax }}$ " and the initial load "P $\mathrm{D}_{\mathrm{D} 0}$ ".

The increase in the power consumed by the load, after the transfer limit point " $P_{\text {Dmax }}$ ", makes the system unstable and provokes a voltage failure. To ensure safety at different system disturbances, the system must operate far from the limit point or point " $\mathrm{C}$ ".

The "V-P" curves can be plotted for different load power factors and to analyze the changes in voltage for different node loadings.

\subsection{Modal analysis}

The modal analysis is based on obtaining values and eigenvectors of the reduced Jacobian, for the voltage stability analysis and the critical points of the network. Positive large eigenvalues mean that the system is stable and far from the limits, whereas positive small eigenvalues represent proximity to the stability limit, and negative eigenvalues represent instability of the EPS [18].

Using modal analysis, the critical points of the network can be obtained by calculating the participation that each element has in each eigenvalue obtained by the reduced Jacobian [18].

\subsubsection{Node participation factor}

The node participation factor in an EPS determines the areas associated with each mode of the system, which are usually of two types. The first type has very few nodes, and they have large participation factors, and the other nodes have participation factors close to zero, which indicates that the mode is very localized in one area. The second type has many nodes with small and similar participation factors, and the remaining nodes have participation factors close to zero, which indicates that the mode is not located in one area. A typical non-localized mode occurs when a single node is connected to a very strong network through a transmission line and the main reactive support of this region has been depleted.

To quantify the relevance of a given node in a specific mode, the node participation factor is defined, which allows identifying the most critical nodes in that mode.

The voltage stability characteristics of a system can be identified by calculating the Jacobian eigenvalues and eigenvectors. Each eigenvalue has associated right and left eigenvectors. The sum of all node participations for each proper mode is equal to one, since the left and right autovectors are normalized.

The node with the highest participation factor for a given mode indicates that this node has the greatest participation in that mode. A negative mode indicates that reagents must be placed in this node and the voltage stability margin must be increased [19].

\subsubsection{Line participation factor}

For each mode, the line with the highest participation factor consumes the most reactive power in response to an incremental change in the reactive load; therefore, it corresponds to the weakest links or is overloaded. Line participation factors are usually used to identify corrective measures to mitigate voltage stability problems and to select contingencies [19].

\subsubsection{Generator participation factor}

The generator participation factor for a mode indicates the reactive power that a generator supplies in response to an incremental change of the reactive load in the system. This factor provides important information with regard to the adequate distribution of reactive reserves among all of the generators, to maintain an adequate voltage stability margin. 
The highest generator participation factor for a mode identifies the generator that supplies the greater reactive power in response to an incremental change in the reactive load in the system. This factor provides important information with regard to the adequate distribution of reactive power reserves among all of the generators, to maintain an adequate voltage stability margin. The identification of critical nodes in the power system and the elements involved are very important for the voltage stability study, since the weak nodes of the network to be compensated can be analyzed, and the possible events that can decrease the voltage stability and cause instability can be evaluated [19].

\section{PMU Placement Methodology}

With the purpose of the PMU placement for voltage stability monitoring, the following methodology is proposed, where the most critical contingencies and nodes, generators and lines with greater participation factors in a voltage stability study are determined. The methodology has the following stages:

\subsection{Input}

In this stage, different elements of the EPS are modeled under normal operating conditions and after contingencies. In other words, the topology of the EPS is entered for both the initial operating condition and after each contingency.

\subsection{Study}

The purpose of this stage is to determine the power flow studies for the initial condition and for each contingency. In addition, voltage stability analysis is performed for all operating conditions of the EPS. The voltage stability study consists of determining the PV curves, V-Q sensitivity analysis and modal analysis, to determine the stability margins, nodes, branches and generators sensitivity to the voltage stability.

\subsection{Placement criteria}

Subsequently, the N-1 critical contingencies that affect the voltage stability are determined, and these contingencies are selected mainly by the initial results of the voltage stability study for the initial condition of the EPS. After performing the studies in the previous stage, the PMUs placement criteria for the selection of the candidate nodes to be installed with PMUs are defined.

These criteria are the results of the study stage, which are the nodes with a small margin of voltage stability, V-Q sensitive nodes, eigenvalues close to zero, and the nodes, lines and generators with high participation factors.

\subsection{Output}

In this stage, the points where the PMUs will be placed are identified to monitor the voltage stability in the EPS.

From the previous stages, the solution is obtained for the PMUs placement problem raised in this study.

Fig. shows in detail the block diagram of the PMUs placement methodology for voltage stability monitoring. The methodology includes four blocks: input, study stage for both the initial operating condition and for $\mathrm{N}-1$ contingencies, stage with the placement criteria and, finally, the output stage where the nodes that require the installation of PMUs for voltage stability monitoring are selected.

The contingencies that are used for the analysis and the selection of the most critical in relation to the voltage stability are selected according to the initial results of the study stage, where the margins, sensitive nodes and participation factors of the nodes, lines and generators are defined. Subsequently, for each contingency, the power flow and voltage stability calculation are performed again. Finally, the methodology delivers the results of the nodes with the PMU placements as output data.



Fig. 2. Block diagram for the PMU placement methodology. 


\section{Case Study}

For the implementation of the methodology proposed in the previous item, the New England 3- node system is chosen, which is commonly used for PMU placement and voltage stability studies and which already has available data on these studies to serve as a comparison with the proposed methodology. In the implementation of this methodology, the results of the critical contingencies defined in other studies, such as [18], [1], [8], [14], [15] and [20], for the selected EPS were also considered. This EPS is a reduction of the New England system in the United States. This system is small, has low chargeability and has sufficient active power to supply the nodes. It has 39 nodes, 46 lines and a maximum reactive capacitive generation of 2870 MVAr to supply the loads and losses of the network. In the original data of this power system, no reactive power limits are defined; therefore, it was necessary to define a low supply capacity, in such a way that the voltage stability margins remained small or critical and to show the methodology effectiveness for low reactive supply systems. The topology of the system shows a loop system, but it has branches that can be isolated when a contingency occurs. For this EPS, simulations of initial conditions, voltage stability analyses and contingencies are performed, as shown below.

\subsection{Initial operating condition}

The conventional Newton Raphson method is used for the power flow calculation.

Table 1 shows the summary of the calculations for different initial operating conditions, to analyze the contingencies.

In the first column of

Table 1, "item", the power generation and demand, active and reactive losses of the network are presented. The second field, "Unit", defines the units of each item. The PF field corresponds to the conventional power flow of the power system, whose reactive power restrictions are only those of the generators. This operating condition can present restriction violations such as voltage and running amps in the lines.

Table 1. Summary of the calculation for the initial operating condition-New England 39-node.

\begin{tabular}{c|c|c}
\hline Item & Unit & PF \\
\hline $\boldsymbol{P}_{\boldsymbol{G}}$ & MW & 6193.261 \\
$\boldsymbol{Q}_{\boldsymbol{G}}$ & MVAr & 1258532 \\
$\boldsymbol{P}_{\boldsymbol{D}}$ & $\mathrm{MW}$ & 6150.50
\end{tabular}

\begin{tabular}{c|c|c}
$\boldsymbol{Q}_{\boldsymbol{D}}$ & MVAr & 1409.50 \\
$\boldsymbol{P}_{\boldsymbol{P}}$ & MW & 42.761 \\
$\boldsymbol{Q}_{\boldsymbol{P}}$ & MVAr & -150.968 \\
\hline
\end{tabular}

Where:

$P_{G}:$ Total generated active power

$Q_{G}$ : Total generated reactive power

$P_{D}$ : Total demanded active power

$Q_{D}$ Total demanded reactive power

$P_{P}$ : Total active power losses

$Q_{P}$ : Total reactive power losses

\subsection{Analysis of contingencies}

For this EPS, the critical contingencies have been selected using a criterion of stability margin reduction greater than $65 \%$ before the contingencies; in other words, in the case of the conventional power flow shown in Table 2, there is an initial margin of $1008.68 \mathrm{MW}$, and its maximum allowed reduction would be $65 \%$ or $353.038 \mathrm{MW}$. These simulations are performed for possible contingencies that could occur in a power system. Table 2 shows the selection of contingencies, for N-1 cases of the New England 39-node system.

The first field of Table 2, "Affected Element", represents the element type isolated after the contingency. The "Nodes" field defines the connection nodes of the affected elements. The load field presents the active and reactive power of the load. The fields "Initial VSM" and "Contingency VSM" define the voltage stability margin in MW, MVAr and the percentage of the initial operating condition before and after the contingency, respectively. The initial margin is obtained by plotting the V-P curve, and it is defined by the maximum chargeability of the node. For example, in the initial condition, the chargeability is $116.4 \%$ for node 29 ; therefore, the initial margin is $16.4 \%$. Finally, the "VSM Reduction" field defines the reduction percentage of the initial voltage stability margin for each contingency, which is also determined from the V-P curves.

These critical contingencies have been selected because they present a reduction in the voltage stability margin greater than the reduction criterion $(65 \%)$, which was taken from [18]. Table 2 shows that some contingencies have equal margins and reduction percentages; the reason is that the contingency disconnects elements that equally affect the voltage stability, as in the case of a generator - transformer group.

Table 2. Results of the N-1 contingency selection - New England 39-node.

\begin{tabular}{c|c|c|c|c|c|c|c|c|c}
\hline \multirow{2}{*}{$\begin{array}{c}\text { Affected } \\
\text { element }\end{array}$} & \multirow{2}{*}{ Nodes } & Load & Load & \multicolumn{3}{|c|}{ Initial Margin } & \multicolumn{2}{c}{ Contingency Margin } & Reduction \\
\cline { 3 - 9 } & & $\mathbf{( M W )}$ & $\mathbf{( M V A r )}$ & $\mathbf{\%}$ & MW & MVAr & $\%$ & MW & VSM (\%) \\
\hline None & - & 6150 & 1409.5 & 16.4 & 1008.60 & 231.158 & - & - & 0 \\
Generator 9 & 38 & 6150 & 1409.5 & 16.4 & 1008.60 & 231.158 & 1.9 & 116.85 & 88.41 \\
Transformer 12 & $29-38$ & 6150 & 1409.5 & 16.4 & 1008.60 & 231.158 & 1.9 & 116.85 & 88.41 \\
Generator 4 & 33 & 6150 & 1409.5 & 16.4 & 1008.60 & 231.158 & 4.9 & 301.35 & 70.12 \\
Transformer 7 & $19-33$ & 6150 & 1409.5 & 16.4 & 1008.60 & 231.158 & 4.9 & 301.35 & 70.12 \\
Generator 5 & 34 & 6150 & 1409.5 & 16.4 & 1008.60 & 231.158 & 5.5 & 338.25 & 66.46 \\
Transformer 8 & $20-34$ & 6150 & 1409.5 & 16.4 & 1008.60 & 231.158 & 5.5 & 338.25 & 66.46 \\
Generator 6 & 35 & 6150 & 1409.5 & 16.4 & 1008.60 & 231.158 & 5.7 & 350.55 & 65.24 \\
Transformer 9 & $22-35$ & 6150 & 1409.5 & 16.4 & 1008.60 & 231.158 & 5.7 & 350.55 & 65.24 \\
\hline
\end{tabular}

\subsection{V-P Curves}

Through these curves, the response of the system for the initial operating condition and the margins after the contingency can be observed. Fig. shows the V-P curve for node 29 of the New England 39-node EPS. To plot this curve, all of the system loads were increased while maintaining the power factor constant, which represents a critical increase in the system's chargeability. In Fig. , the V-P curves are shown 
for node 29, before and after the contingency. This node was selected because it clearly represents the increase in the margin while maintaining high voltage levels and without presenting voltage violations.

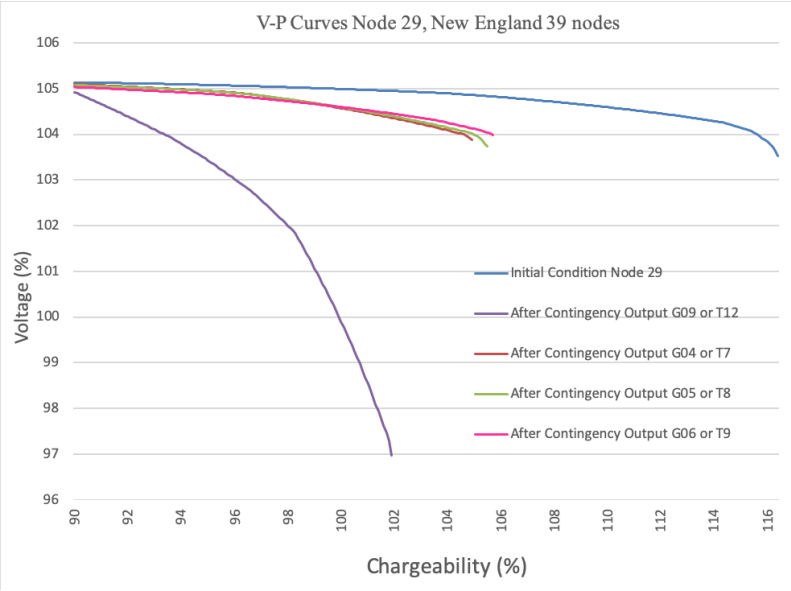

Fig. 3. V-P Curves of node 29-New England 39-node.

Table 3 shows the voltage stability margins for the critical contingencies and for the initial operating conditions.

Table 3. Voltage stability margins - New England 39-node.

\begin{tabular}{|c|c|c|c|c|c|}
\hline \multirow[t]{2}{*}{$\begin{array}{l}\text { Initial } \\
\text { case }\end{array}$} & \multirow[t]{2}{*}{ Event } & \multicolumn{2}{|c|}{$\begin{array}{l}\text { Initial } \\
\text { margin }\end{array}$} & \multicolumn{2}{|c|}{$\begin{array}{l}\text { Contingency } \\
\text { margin }\end{array}$} \\
\hline & & $\%$ & MW & $\%$ & MW \\
\hline \multirow[t]{4}{*}{$\begin{array}{l}\text { Power } \\
\text { flow }\end{array}$} & $\begin{array}{l}\text { Gen } 9 \text { and } \\
\text { Tran } 12\end{array}$ & 16.4 & 1008.6 & 1.9 & 116.85 \\
\hline & $\begin{array}{l}\text { Gen } 4 \text { and } \\
\text { Tran } 7\end{array}$ & 16.4 & 1008.6 & 4.9 & 301.35 \\
\hline & $\begin{array}{l}\text { Gen } 5 \text { and } \\
\text { Tran } 8\end{array}$ & 16.4 & 1008.6 & 5.5 & 338.25 \\
\hline & $\begin{array}{l}\text { Gen } 6 \text { and } \\
\text { Tran } 9\end{array}$ & 16.4 & 1008.6 & 5.7 & 350.55 \\
\hline
\end{tabular}

\subsection{V-Q Sensitivity analysis}

The V-Q sensitivity analysis was performed at the New England 39-node EPS. Fig. shows the results for the initial operating condition and after the output contingency of Generator 9, which is the most critical. The higher the V-Q Sensitivity value (\%/MVAr) is, the closer to the stability limit. Moreover, small sensitivity values represent higher stability of the system. Nodes 12 and 28 show the highest V-Q sensitivity for the initial condition, and then, after the G9 output, which is the most critical contingency, nodes 29, 28, 12 and 26 have the highest voltage sensitivity with regard to the reactive power change.

Comparison of the V-Q Sensitivity analysis New England 39 nodes

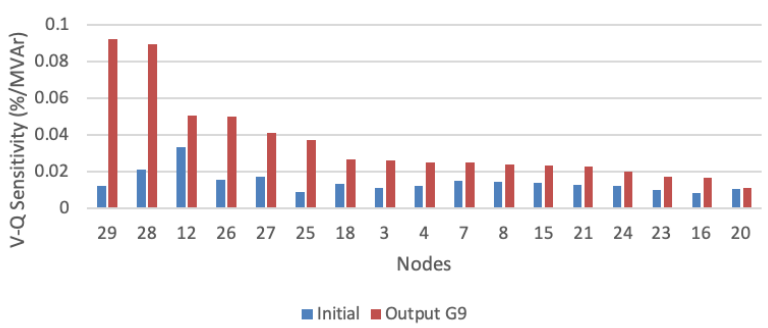

Fig. shows the results for the initial operating condition and after all of the contingencies identified as critical are determined. This figure shows that nodes 12 and 28 have the highest V-Q sensitivity for the initial condition, and then, after the contingencies, nodes 29, 28, 12, 26, 27, 25 and 20 have the highest voltage sensitivity with regard to the reactive power change.

\section{9-node.}

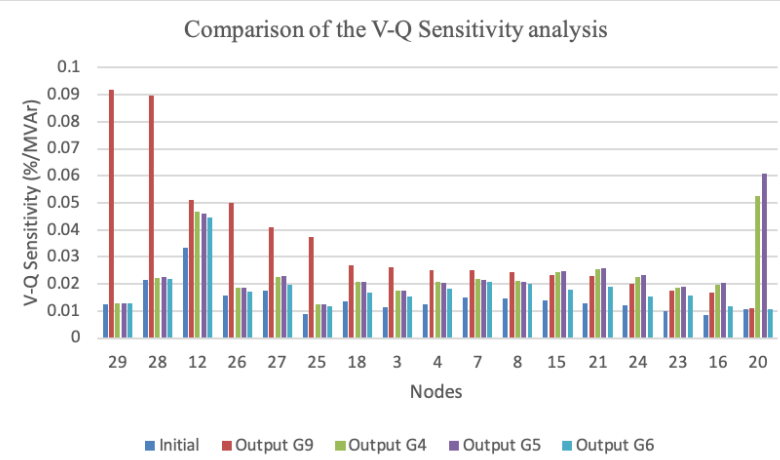

Fig. 5. Comparison of V-Q sensitivity analysis - New England

Table 4 shows the voltage sensitivity comparison of the nodes before the change in the reactive power for the New England 39-node EPS. It is presented for the initial operating condition and after the output contingency of the Generator 9 located in node 38 .

Table 4 .V-Q Sensitivity - New England 39-node.

\begin{tabular}{c|c|c}
\hline $\begin{array}{c}\text { Nod } \\
\mathbf{e}\end{array}$ & $\begin{array}{c}\text { Initial Condition } \\
\text { \%/MVAr }\end{array}$ & $\begin{array}{c}\text { After } \\
\text { Contingency } \\
\text { \%/MVAr }\end{array}$ \\
\hline $\mathbf{2 9}$ & 0.012574 & 0.09199 \\
$\mathbf{2 8}$ & 0.021481 & 0.089703 \\
$\mathbf{1 2}$ & 0.033223 & 0.050897 \\
$\mathbf{2 6}$ & 0.015617 & 0.050129 \\
$\mathbf{2 7}$ & 0.017545 & 0.041087 \\
$\mathbf{2 5}$ & 0.009029 & 0.037491 \\
$\mathbf{1 8}$ & 0.013512 & 0.026899 \\
$\mathbf{3}$ & 0.01131 & 0.026265 \\
$\mathbf{4}$ & 0.012553 & 0.025163 \\
$\mathbf{7}$ & 0.014981 & 0.025146 \\
$\mathbf{8}$ & 0.014635 & 0.024241 \\
$\mathbf{1 5}$ & 0.013793 & 0.023279 \\
$\mathbf{2 1}$ & 0.012795 & 0.023094 \\
$\mathbf{2 4}$ & 0.012203 & 0.019994 \\
$\mathbf{2 3}$ & 0.010003 & 0.017511 \\
$\mathbf{1 6}$ & 0.008666 & 0.016714 \\
$\mathbf{2 0}$ & 0.010567 & 0.010956 \\
\hline
\end{tabular}

\subsection{Modal analysis - Participation factors}

The closer the eigenvalue is to zero, the closer the system will be to voltage instability. Fig shows the eigenvalues of the New England EPS for a normal operating condition and after the critical contingency. This simulation is conducted while considering all of the contingencies that are identified as critical.

Figure 6 shows the EPS with an initial operating condition that is far from the voltage instability. Once the contingencies occur, the eigenvalues are considerably reduced and approach zero.

Fig. 4. V-Q Sensitivity analysis - New England 39-node. 


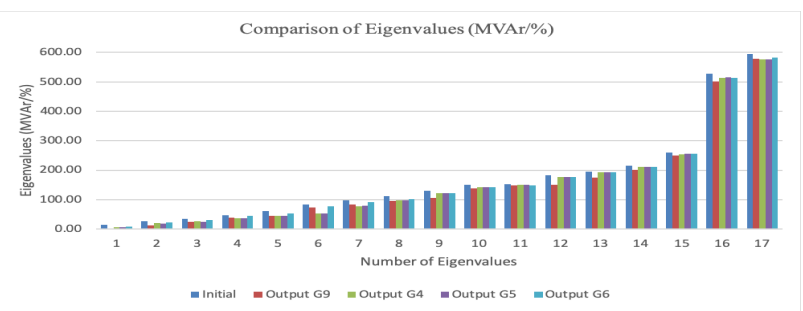

Table 5 presents comparison data of the eigenvalues of the system for the initial condition and for the most critical contingencies of the system.

The "Initial" field defines the eigenvalues for the operation of the system before the contingencies. The Table 5. Eigenvalues - New England 39-node.

\begin{tabular}{c|c|c|c|c|c}
\hline \multicolumn{7}{c}{ Eigenvalues MVAr/\% } & \multicolumn{1}{c}{ Output G5 } & Output G6 \\
\hline Eigenvalue & Initial & Output G9 & Output G4 & 5.47 & 8.97 \\
\hline $\mathbf{1}$ & 15.30 & 2.94 & 5.70 & 19.38 & 21.97 \\
$\mathbf{2}$ & 25.95 & 11.64 & 19.66 & 25.18 & 30.77 \\
$\mathbf{3}$ & 35.72 & 24.20 & 25.77 & 36.21 & 44.38 \\
$\mathbf{4}$ & 47.29 & 38.17 & 37.49 & 44.93 & 53.71 \\
$\mathbf{5}$ & 60.37 & 44.97 & 44.67 & 53.87 & 78.34 \\
$\mathbf{6}$ & 82.56 & 72.75 & 53.78 & 78.46 & 92.46 \\
$\mathbf{7}$ & 97.59 & 83.43 & 78.32 & 121.18 & 101.40 \\
$\mathbf{8}$ & 111.99 & 96.14 & 96.80 & 142.88 & 121.15 \\
$\mathbf{9}$ & 130.37 & 105.73 & 121.05 & 149.58 & 142.48 \\
$\mathbf{1 0}$ & 149.46 & 138.50 & 142.32 & 193.41 & 148.65 \\
$\mathbf{1 1}$ & 152.13 & 148.90 & 149.71 & 212.17 & 177.48 \\
$\mathbf{1 2}$ & 182.79 & 149.91 & 177.26 & 255.36 & 193.89 \\
$\mathbf{1 3}$ & 194.75 & 175.61 & 193.83 & 516.40 & 212.19 \\
$\mathbf{1 4}$ & 215.81 & 201.90 & 212.03 & 576.56 & 255.03 \\
$\mathbf{1 5}$ & 260.34 & 249.34 & 254.85 & 513.08 & 512.91 \\
$\mathbf{1 6}$ & 527.52 & 502.27 & 577.12 & & 583.31 \\
\hline
\end{tabular}

Fig. 6. Eigenvalues - New England 39-node.

"Output" field defines the eigenvalues for the operation of the system after each critical contingency.
Table 6 shows the comparison of eigenvalues for each operating condition in which the nodes are identified with their corresponding participation factor. This comparison allows us to identify the nodes that have the highest participation in each eigenvalue, and a low eigenvalue indicates a proximity to the voltage stability limit.

Table 6. Comparison of eigenvalues for each operating condition - New England 39-node.

\begin{tabular}{|c|c|c|c|c|c|c|c|c|c|}
\hline \multirow{2}{*}{\multicolumn{2}{|c|}{$\begin{array}{c}\text { Initial condition } \\
\text { Eigenvalue } \\
15.3 \\
\end{array}$}} & \multirow{2}{*}{\multicolumn{2}{|c|}{\begin{tabular}{|c|} 
Output G9 \\
Eigenvalue \\
2.94 \\
\end{tabular}}} & \multirow{2}{*}{\multicolumn{2}{|c|}{\begin{tabular}{|c|} 
Output G4 \\
Eigenvalue \\
5.70 \\
\end{tabular}}} & \multirow{2}{*}{\multicolumn{2}{|c|}{$\begin{array}{c}\text { Output G5 } \\
\text { Eigenvalue } \\
5.47 \\
\end{array}$}} & \multirow{2}{*}{\multicolumn{2}{|c|}{$\begin{array}{c}\text { Output G6 } \\
\text { Eigenvalue } \\
8.97 \\
\end{array}$}} \\
\hline & & & & & & & & & \\
\hline Nodes & Factor & Nodes & Factor & Nodes & Factor & Nodes & Factor & Nodes & Factor \\
\hline 12 & 0.1211 & 29 & 0.2057 & 20 & 0.1323 & 20 & 0.1663 & 12 & 0.1628 \\
\hline 27 & 0.1014 & 28 & 0.2023 & 15 & 0.0932 & 15 & 0.0916 & 4 & 0.0923 \\
\hline 18 & 0.0855 & 26 & 0.1203 & 16 & 0.0882 & 16 & 0.0895 & 7 & 0.0834 \\
\hline 15 & 0.0813 & 27 & 0.0888 & 12 & 0.0863 & 24 & 0.0855 & 15 & 0.0819 \\
\hline 26 & 0.0749 & 25 & 0.0671 & 24 & 0.0845 & 21 & 0.0804 & 8 & 0.0796 \\
\hline 4 & 0.0716 & 18 & 0.0475 & 21 & 0.0798 & 12 & 0.0764 & 18 & 0.0718 \\
\hline 24 & 0.0634 & 3 & 0.0459 & 18 & 0.0687 & 18 & 0.0667 & 3 & 0.0651 \\
\hline 16 & 0.0634 & & & 4 & 0.0623 & 4 & 0.0567 & 27 & 0.0611 \\
\hline 7 & 0.0622 & & & 27 & 0.0553 & 27 & 0.0538 & 16 & 0.0598 \\
\hline 3 & 0.0619 & & & 3 & 0.0514 & 3 & 0.0486 & 24 & 0.0597 \\
\hline 8 & 0.0602 & & & 7 & 0.0467 & 23 & 0.0412 & 21 & 0.0591 \\
\hline 28 & 0.0479 & & & 8 & 0.0446 & 7 & 0.0411 & 26 & 0.0413 \\
\hline 21 & 0.0443 & & & 23 & 0.0410 & 8 & 0.0392 & & \\
\hline & & & & 26 & 0.0327 & & & & \\
\hline
\end{tabular}

Fig. shows the node participation factor for the initial operating condition of the New England 39-node EPS. In this figure, it is identified that the nodes with the highest participation are nodes 12,27 and 18 . 
Initial Condition of New England 39-node Eigenvalue 15.3

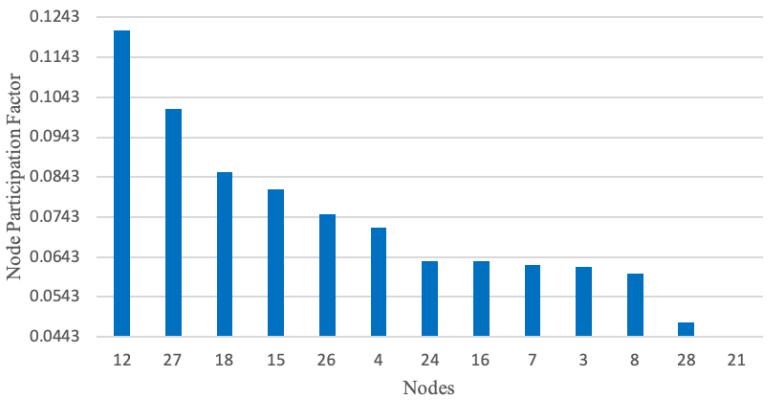

Fig. 7. Node participation factor for the initial operating condition - New England 39-node

Fig. 2 shows the node participation factor for the critical contingency output of generator 9 for the New England 39node system. In this figure, it is identified that the nodes with the highest participation are nodes 29,28 and 26, and it is confirmed that node 29 is one of the most sensitive in relation to the $\mathrm{V}-\mathrm{Q}$.

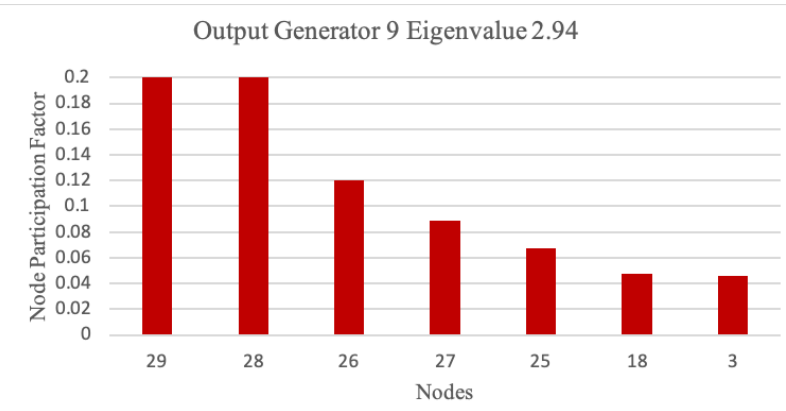

Fig. 2. Node participation factor for critical contingency - New England 39-node

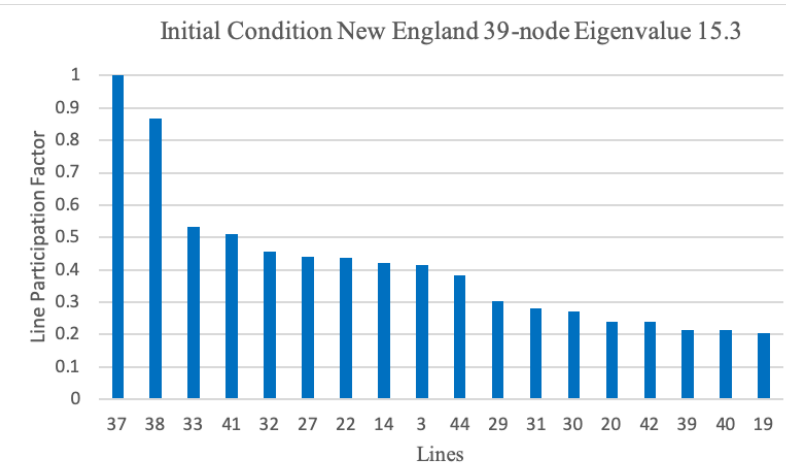

Fig. 3 shows the line participation factor to determine the most sensitive and overloaded for the initial operating condition of the New England 39-node EPS. As a result, the most sensitive lines are 37, 38, 33 and 41 . Lines 37 and 38 are transformers. Line 37 connects nodes 6 and 31, line 38 connects nodes 10 and 32 , line 33 connects nodes 26 and 29, and finally, line 41 connects nodes 22 and 35 .
Initial Condition New England 39-node Eigenvalue 15.3

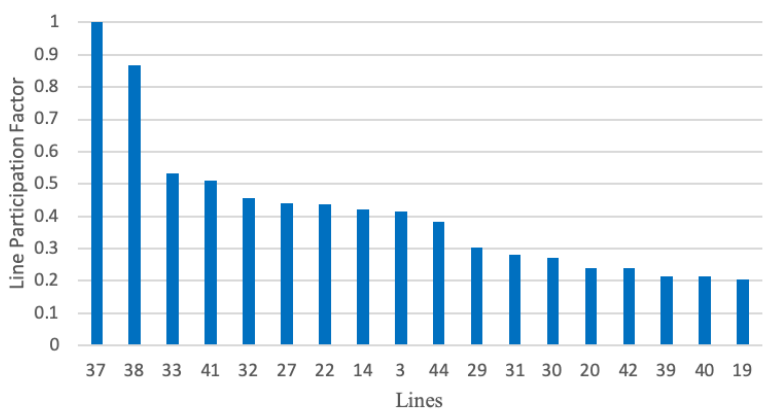

Fig. 3. Line participation factor for initial operating condition - New England 39-node

Fig. 4 shows the line participation factor for the generator 9 output of the New England 39-node system. In this figure, it is identified that the lines with the highest participation are 37, 33 and 32, which are located between nodes 6-31, 26-29 and $26-28$ respectively.

Output Generator 9 New England 39-node Eigenvalue 2.94

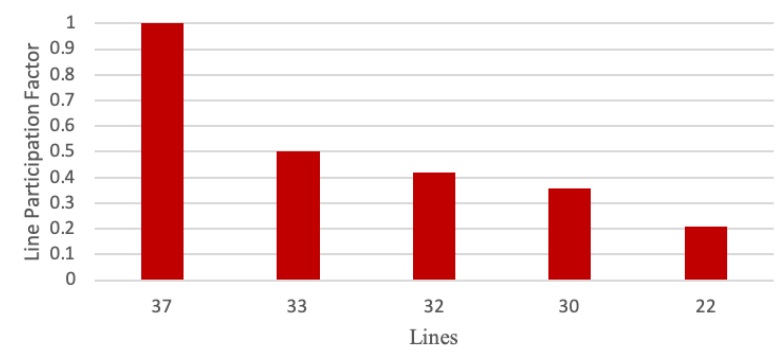

Fig. 4. Line participation factor for critical contingency - New England 39-node

Fig. 5 shows the generator participation factor for the initial operating condition of the New England 39-node EPS. In this figure, it is shown that the generators with the lowest reactive reserve are those located in nodes 9, 3, 6 and 2 .

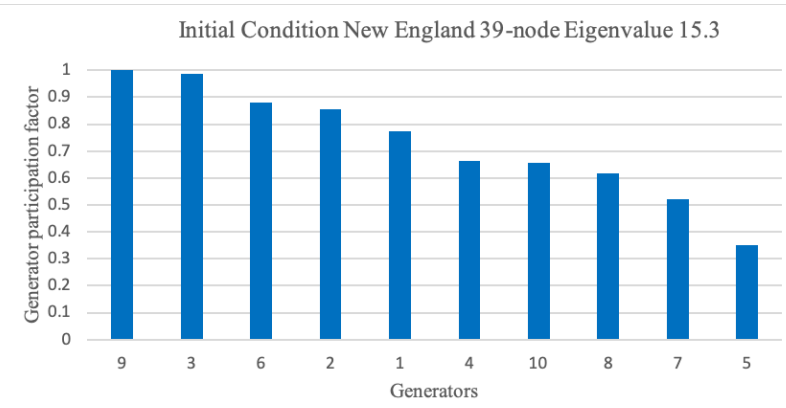

Fig. 51. Generator participation factor for initial operating condition New England 39-node

Fig. 6 shows the generator participation factor for the generator 9 output of the New England 39-node system. In this figure, it is shown that the generator with the highest participation for this event is the generator located in node 38 . 




Fig. 62. Generator participation factor for critical contingency - New England 39-node

\subsection{PMU placement nodes.}

According to the stability analysis of the static voltage performed on the previous items, the PMU placement nodes are presented in

Table 7.

Table 7. PMUs placement nodes - New England 39-node.

\begin{tabular}{c|c}
\hline Nodes & Criteria \\
\hline $\mathbf{1 2}$ & VQ Sensitivity, Node participation factor \\
$\mathbf{1 5}$ & VQ Sensitivity, Node participation factor \\
$\mathbf{1 8}$ & VQ Sensitivity, Node participation factor \\
$\mathbf{2 0}$ & VQ Sensitivity, Node participation factor \\
$\mathbf{2 5}$ & VQ Sensitivity, Node participation factor \\
$\mathbf{2 6}$ & VQ Sensitivity, Node participation factor
\end{tabular}

VQ Sensitivity, Node participation factor

VQ Sensitivity, Node participation factor VQ Sensitivity, PV Curve, Node participation factor

\section{Conclusions}

In this article, a PMU placement methodology for voltage stability monitoring was presented. The methodology uses V$\mathrm{Q}$ sensitivity criteria and considers the V-P curves to determine the voltage stability margins and the participation factors of the nodes, lines and generators. Finally, because the voltage stability monitoring implies that the system works under different operating scenarios, the N-1 output lines of the system were also included. The proposed PMU placement methodology also considers the N-1 contingencies, which changes the topology of the system. The methodology was tested in the New England 39-node system for the placement of PMUs for voltage stability monitoring, and the results ensure that the nodes for the PMU placements are the most sensitive to the voltage stability problems under normal operating conditions and under critical contingencies.

This is an Open Access article distributed under the terms of the Creative Commons Attribution License

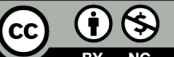

\section{References}

[1] R. Sodhi, S. C. Srivastava, and S. N. Singh, "Optimal PMU Placement to Ensure System Observability under Contingencies," Power Energy Soc. Gen. Meet. 2009. PES '09. IEEE, pp. 1-6, 2009.

[2] P. Zhang, "Phasor Measurement Unit ( PMU ) Implementation and Applications," Electr. Power Res. Inst. EPRI, Rep. Final 2007., 2007.

[3] Z. Hong-Shan, L. Ying, M. Zeng-qiang, and Y. Lei, "Sensitivity Constrained PMU Placement for Complete Observability of Power Systems," Transm. Distrib. Conf. Exhib. Asia Pacific, 2005 IEEE/PES, pp. 1-5, 2005.

[4] J. Peng, Y. Sun, and H. Wang, "Optimal PMU placement for full network observability using Tabu search algorithm," Electr. Power Energy Syst., vol. 28, no. 4, pp. 223-231, May 2006.

[5] G. N. Korres, P. S. Georgilakis, N. C. Koutsoukis, and N. M. Manousakis, "Numerical observability method for optimal phasor measurement units placement using recursive Tabu search method," IET Gener. Transm. Distrib., vol. 7, no. 4, pp. 347-356, Apr. 2013.

[6] H. Mesgarnejad and S. M. Shahrtash, "Multi-objective measurement placement with new parallel Tabu Search method," in 2008 IEEE Canada Electric Power Conference, 2008, pp. 1-6.

[7] A. Gamm, I. Kolosok, A. Glazunova, and E. Korkina, "PMU placement criteria for EPS state estimation," Electr. Util. Deregul. Restruct. Power Technol. 2008. DRPT 2008. Third Int. Conf., pp. 645-649, 2008.

[8] B. Milosevic and M. Begovic, "Nondominated Sorting Genetic Algorithm for Optimal Phasor Measurement Placement," Power Syst. IEEE Trans., vol. 18, no. 1, pp. 69-75, 2003.

[9] H. G. and H. S. M. Farsadi, "Phasor Measurement Unit (PMU) allocation in power system with different algorithms," in 2009 International Conference on Electrical and Electronics Engineering - ELECO 2009, 2009, pp. 396-400.

[10] T.-T. Cai and Q. Ai, "Research of PMU Optimal Placement in Power Systems," in 2005 World Scientific and Engineering Academy and Society Int. Conf, 2005, pp. 38-43.
[11] R. F. Nuqui, A. G. Phadke, and L. Fellow, "Phasor Measurement Unit Placement Techniques for Complete and Incomplete Observability," Power Deliv. IEEE Trans., vol. 20, no. 4, pp. 2381-2388, 2005.

[12] B. Gou, "Optimal Placement of PMUs by Integer Linear Programming," Power Syst. IEEE Trans., vol. 23, no. 3, pp. 15251526, 2008.

[13] B. Xu and A. Abur, "Observability Analysis and Measurement Placement for Systems with PMUs," Power Syst. Conf. Expo., vol. 2, pp. 943-946, 2004.

[14] K. J. Makasa and G. K. Venayagamoorthy, "On-line Voltage Stability Load Index Estimation Based on PMU Measurements," Power Energy Soc. Gen. Meet. 2011 IEEE, pp. 1-6, 2011.

[15] R. F. Nuqui, A. G. Phadke, R. P. Schulz, and N. Bhatt, "Fast online voltage security monitoring using synchronized phasor measurements and decision trees," in 2001 IEEE Power Engineering Society Winter Meeting. Conference Proceedings (Cat. No.01CH37194), 2001, vol. 3, pp. 1347-1352.

[16] G. B. Denegri, M. Invernizzi, F. Milano, M. Fiorina, and P. Scarpellini, "A Security Oriented Approach to PMU Positioning for Advanced Monitoring of a Transmission Grid," Power Syst. Technol. 2002. Proceedings. PowerCon 2002. Int. Conf., vol. 2, pp. 798-803, 2002.

[17] S. Chakrabarti and E. Kyriakides, "Optimal Placement of Phasor Measurement Units for Power System Observability," Power Syst. IEEE Trans., vol. 23, no. 3, pp. 1433-1440, 2008.

[18] J. E. Candelo Becerra, "Metodología para mejorar la estabilidad de voltaje mediante la coordinación de Facts y compensadores de reactivos suicheados mecánicamente," Universidad del Valle, 2008.

[19] G. N. Caicedo Delgado, Estabilidad en sistemas eléctricos de potencia, Primera Ed. Cali Colombia, 2018.

[20] S. M. Pérez Londoño, "Propuesta para la identificación y monitoreo de áreas débiles con relación a la estabilidad de tensión en sistemas eléctricos de potencia," Universidad Nacional de Colombia, 2013. 